Document downloaded from:

http://hdl.handle.net/10251/107463

This paper must be cited as:

Rubio Michavila, C.; Castiñeira Ibáñez, S.; Uris Martínez, A.; Belmar Ibáñez, F.; Candelas Valiente, P. (2018). Numerical simulation and laboratory measurements on an open tunable acoustic barrier. Applied Acoustics. 141:144-150. doi:10.1016/j.apacoust.2018.07.002

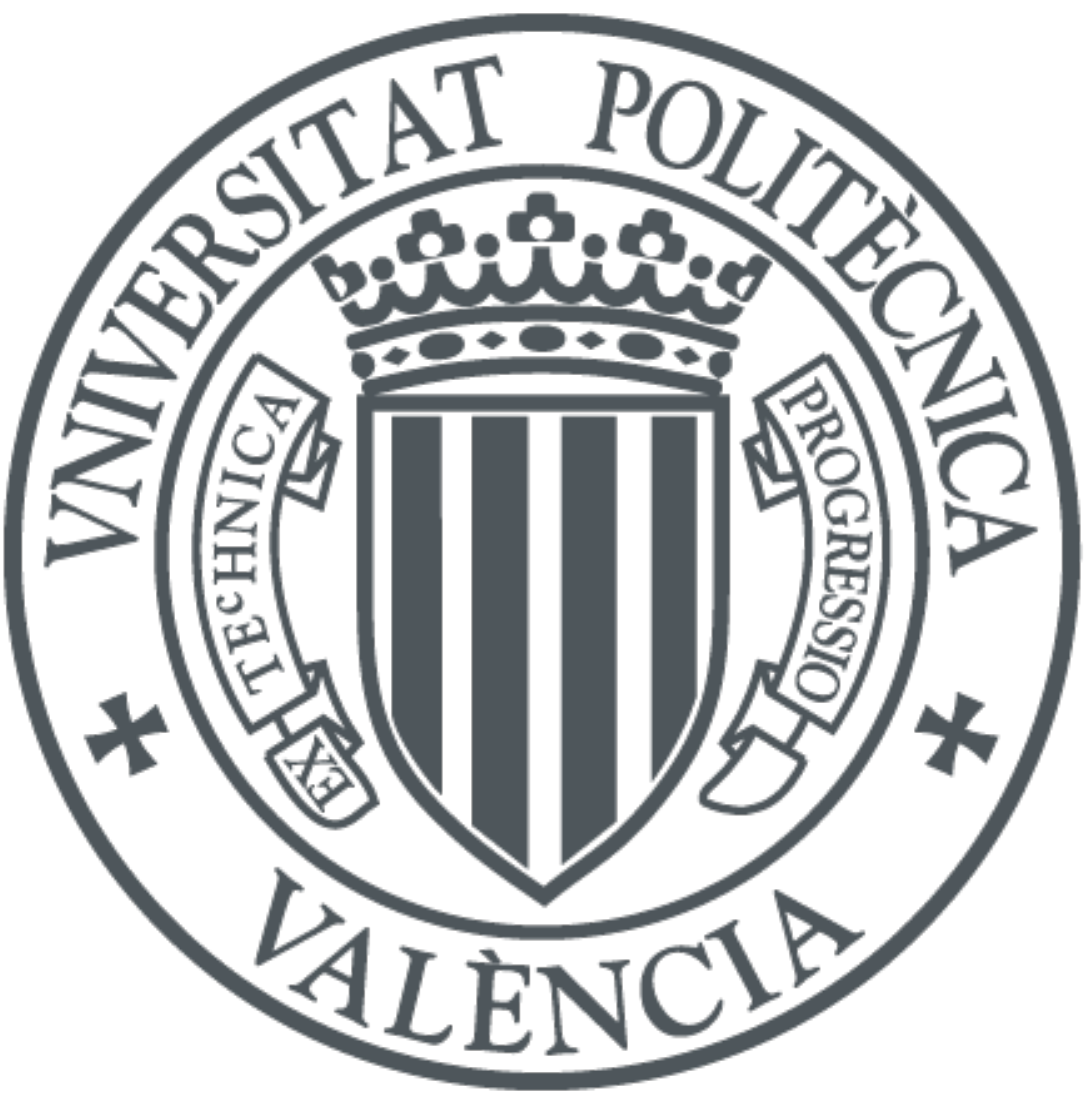

The final publication is available at

https://doi.org/10.1016/j.apacoust.2018.07.002

Copyright Elsevier

Additional Information 


\title{
NUMERICAL SIMULATION AND LABORATORY MEASUREMENTS ON AN OPEN TUNABLE ACOUSTIC BARRIER
}

\author{
Constanza Rubio*, Sergio Castiñeira-Ibáñez, Antonio Uris, Francisco Belmar, Pilar Candelas \\ Centro de Tecnologías Físicas: Acústica, Materiales y Astrofísica. División Acústica. \\ Universitat Politécnica de Valencia. Camino de Vera s/n. 46022 Valencia, Spain. \\ Phone: 34.963877528; Fax: 34.963879525; E-mail: crubiom@ fis.upv.es \\ *Corresponding author.
}

\begin{abstract}
A new open, thin and low frequency acoustic barrier is presented. These barriers, based on arrays of isolated pickets produce high acoustic attenuation in a selective range of frequencies related to their geometry and distribution. These open barriers are acoustically competitive with traditional ones, which are based on continuous and rigid materials. To show its versatility t $\Theta$ in attenuating different selected ranges of frequencies, a compact numerical model is presented.

Different cases are analysed and compared with experimental results. The accuracy of the experimental results compared to the simulated ones allow us to use the compact model to design these barriers in order to reduce both industrial and traffic noise on demand and to introduce them into the noise control market.
\end{abstract}

Keywords: Acoustic barrier, insertion loss, acoustic quarter subwavelength resonator, industrial noise, building protection. 


\section{INTRODUCTION}

To reduce or eliminate the discomfort originated by noise, modifications can be carried out on the sound source, on the medium of propagation or on the receiver. When an object is interposed in the path of sound propagation between the source and the receiver, that object can act as an acoustic barrier, causing a decrease in the sound level at the position of the receiver. It is in this action framework in noise control where this paper is found. Although research on acoustic barriers has been developing since 1950, the scientific community remains active in this area. The potential for noise attenuation of acoustic barriers with absorbent surfaces has also been evaluated. The research has mainly focused on applications with parallel displays on both sides of a road, since reflected noise between barriers is believed to increase noise levels $[1,2,3]$. The intrinsic acoustic characteristics of installed noise barriers, such as sound reflection, have also been checked to verify their compliance to design specifications or their quality after some years of life [4]. However, these traditional acoustic barriers are continuous vertical walls that must be weighed and be very tall in order to attenuate low frequency noise. In addition, these types of continuous barriers block the flow of air and water, which may limit their applications [5].

In the last two decades, there has been a growing interest in the research of periodic acoustic barriers. The major benefit of these barriers is that they produce high attenuation in selective ranges of frequencies depending on their geometry, that is, they can be frequency tuneable. Open acoustic barriers have other benefits such as they are permeable to wind and water. Moreover, they have an aesthetically pleasing appearance. These facts make them ideal both for use in roads, industrial facilities and naturally ventilated buildings. One of these types of open barriers is sonic crystal barriers, that are composite structures with their dispersing elements arranged periodically. In fact, the presence of 
acoustic dispersers in the medium with different acoustics characteristics, leads to the appearance of frequency intervals, dictated by Bragg's law, in which the acoustic waves do not propagate, being on the contrary totally reflected. The efficiency of the use of sonic crystal in the design of acoustic barriers with cylindrical dispersers has been demonstrated in different works $[6,7]$ and an example of these sonic crystal acoustic barriers can be seen in the A2 ring road of Eindhoven where Van Campen industries installed 22,000 $\mathrm{m}^{2}$ of them [8]. Other types of open acoustic barriers have also been developed, different from those based on sonic crystals, that are periodic and frequency tuneable [9]. These barriers are based on a periodic arrangement of subwavelength slits created by rectangular pickets distributed periodically.

In this paper, a solution for low frequency noise mitigation based on a periodic structure and acoustic resonators is proposed. The idea of combining the effects of periodicity and resonators aims to achieve noise attenuation at the low frequency range. This new acoustic barrier design is frequency tuneable and versatile, providing a simple design that could represent a good option for managing low frequency noise in buildings. It could also be used both for the protection of buildings against traffic noise and for the protection of buildings against industrial or for equipment noise. In fact, this Open and Tunable Acoustic Barrier (OTAB) is integrated by pickets that can be designed to choose the ranges of frequencies in which the barrier must act. This action framework makes these barriers technologically advanced. Moreover, the pickets includes different noise control mechanism such as absorption or resonances so that the designer could select which of these mechanisms should appear and at which frequency it should work (following the concept of tuneability) [10].

The great advantage of the OTAB in contrast to previously existing open acoustic barriers, is its thickness. To the best of our knowledge the acoustic barriers based on sonic 
crystals have to be at least $0.60 \mathrm{~m}$ thick, which limits and precludes its use in certain applications. The acoustic behaviour of this barrier has been analysed through both numerical modelling and experimental testing under laboratory conditions where it could be verified that this OTAB, with a thickness of $0.30 \mathrm{~m}$, has a great attenuation capacity at low frequencies.

\section{SUBWAVELENGTH SLIT ACOUSTIC BARRIER}

The basic structure of a subwavelength slit acoustic barrier consists of one or two rows of rectangular pickets distributed periodically with a lattice period $l_{p}$ and with a slit width $\mathrm{s}_{\mathrm{w}}$. The rows are separated by an air gap $d_{\mathrm{ag}}$ as shown in Figure 1. The slit width is $\mathrm{s}_{\mathrm{w}}$ and is much less than the wavelength, $\lambda$.

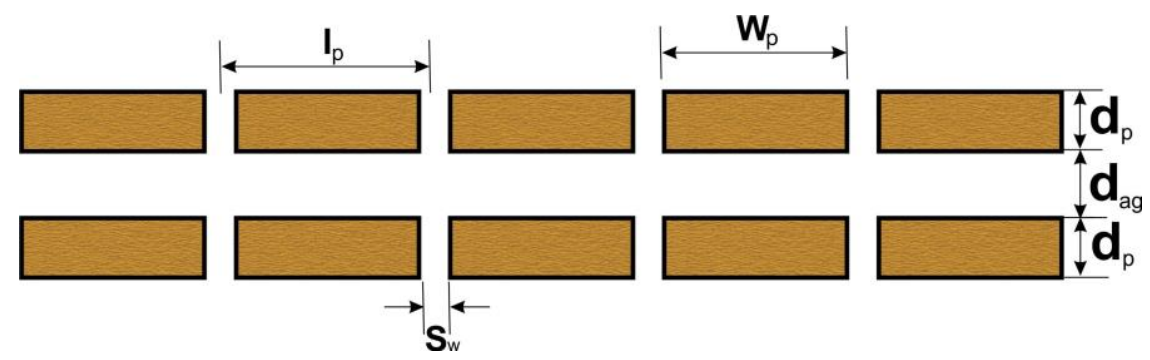

Figure 1. Schematic diagram of a basic subwavelength slit acoustic barrier.

Several mechanism and physical phenomena contribute to the acoustic attenuation of this type of barrier. In the first place, and due to the configuration and distribution of the pickets, the acoustic barrier can be considered to be formed by a series of acoustic filters. The slits act as inlet/outlet apertures and the air gap between the picket rows acts as an expansion chamber. Moreover, the geometric parameters of the acoustic barrier play an important role in acoustic attenuation. Since the pickets are distributed periodically with a lattice period $l_{\mathrm{p}}$, some acoustic attenuation peaks appear in the attenuation spectrum, at certain frequencies, which are the manifestation of Wood's anomalies [11]. For normal incidence, Wood's anomaly appears when the wavelength of the incident wave coincides 
with the lattice period, $\lambda=l_{\mathrm{p}}$. On the other hand, attenuation peaks also appear in midrange frequencies that are due to the interferences between propagating and evanescent waves [12]. A complete analysis of this type of acoustic barrier can be found in [9].

\section{NUMERICAL APPROACH: FINITE ELEMENT METHOD}

The geometry shown in Figure 2a has been defined to solve the problem numerically. We have designed an acoustic barrier with 2 rows following the guidelines proposed by [9] in order to obtain a high level of attenuation. The commercial software COMSOL Multiphysics has been used to design the barriers, to develop the model and to obtain the numerical predictions. The domain where the solution is obtained is formed by 2 pickets with the following dimensions: width $0.3 \mathrm{~m}\left(\mathrm{w}_{\mathrm{p}}\right)\left(\mathrm{X}\right.$ direction) and depth $0.1 \mathrm{~m}\left(\mathrm{~d}_{\mathrm{p}}\right)(\mathrm{Y}$ direction), separated along $\mathrm{Y}$ direction by an air gap $\left(\mathrm{d}_{\mathrm{ag}}\right)$ of $0.1 \mathrm{~m}$, and confined between two completely reflected walls separated by $0.35 \mathrm{~m}$ (picket width plus subwavelenght slit, $S_{\mathrm{w}}=0.05 \mathrm{~m}$ ) as shown in Figure $2 \mathrm{~b}$, the $\mathrm{Y}$ axis being the propagation direction of the incident plane wave (IPW) travelling from left to right. With these conditions, the scattered waves from the pickets are reflected by the walls reproducing the effect of a semiinfinite barrier, formed by 2 rows of pickets with a distance between them of $0.35 \mathrm{~m}$ along the $\mathrm{X}$ direction $\left(l_{\mathrm{p}}\right.$ ), and whose centres are separately by $0.2 \mathrm{~m}$ (air gap plus two half lengths of the pickets along $\mathrm{Y}$ direction, $\mathrm{d}_{\mathrm{ag}}+2 \mathrm{~d}_{\mathrm{p}} / 2$ ). Moreover, the walls do not reflect the IPW. This geometry allows us to understand the physical phenomena of semiinfinite arrays using a reduced volume of the numerical domain thus diminishing the burden of the Finite Element Method (FEM). Two boundaries of the domain are selected as a periodic condition which is a good alternative to simulate an infinite long barrier in $\mathrm{X}$ direction, whilst the other two are considered a radiation boundary condition in order 
to implement the Sommerfeld radiation conditions in the numerical resolution of scattering problems and therefore to simulate free space. The last domain is implemented with Perfectly Matched Layers (PML) [13], to avoid the return of the waves to the main domain.

Firstly, the pickets in question include several physics mechanism such as acoustic filter and resonances whose section is shown in Figure $2 \mathrm{a}$. When all the surfaces of each of the pickets are acoustically rigid, the Neumann boundary condition (zero sound velocity) is applied to them and they act as an acoustic filter due to the fact that a change in section occurs between the two rows. If one of their surfaces is enabled and the others remain rigid, a cavity is obtained and they act as a quarter-wavelength resonator ( $\lambda / 4$ resonator). From these pickets and with a view to improving the attenuation of the barrier, a numerical design is presented that is more sophisticated than the previous one and at the same time is simpler to modify. These new pickets are based on the activation and deactivation of the different layers depending on the desired effect. We call this model a compact model.

These new designs that introduce more acoustic mechanisms to the picket can be considered as multiphenomenon pickets (MP). Thus, a layer of absorbing material is introduced around the picket keeping a thin layer of material that provides the rigidity of the picket, forcing it to keep its original size. The interior of the cavity can be completely filled with air or to tuned to another resonance frequency, by reducing the size of the cavity with the activation of a rigid block, see Figure $2 b$. In order to achieve it, the pickets were implemented as shown in Figure $2 \mathrm{~b}$. The MP are a rigid rectangle covered by a porous material. Neumman boundary condition is applied to the rigid surfaces as mentioned above, whereas the Delany-Bazley [14] model is considered to emulate the porous material. The cavity is considered filled with air, thus the length of the resonator 
defines the fundamental resonant frequency and its harmonics, given by the expression [15]:

$$
f=\frac{c}{L\left(\frac{2 n+1}{4}\right)} \quad n=0,1,2 \ldots
$$

where $\mathrm{c}$ is sound velocity $(\mathrm{c}=340 \mathrm{~m} / \mathrm{s})$ and $\mathrm{L}$ is the length of the resonator cavity. When $\mathrm{n}=0 \mathrm{~L}$ is equal to $\lambda / 4$. With these conditions by enabling or disabling the surfaces, it is easier and more suitable to select the desired behaviour and the acting frequency of the OTAB

(a)

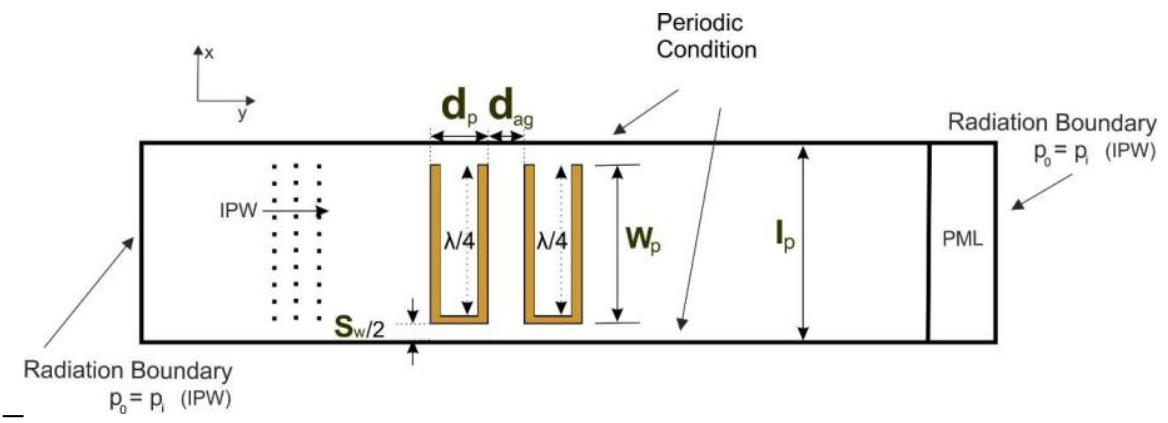

(b)

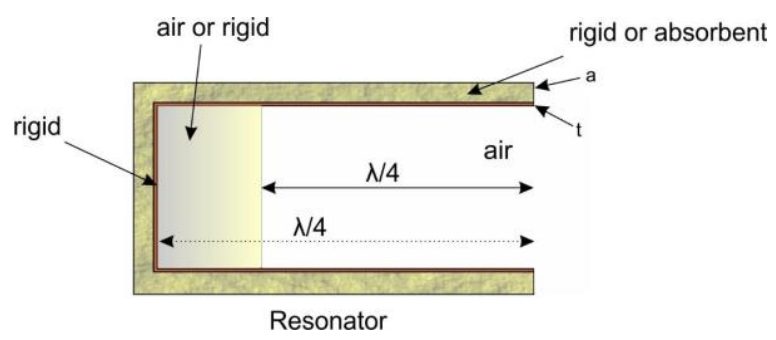

Figure 2.- Design of the ideal numerical model: (a) Plan view (OXY, two dimensional case), which includes resonances and acoustic filter. One can see the OXY section of the multi-phenomena pickets, as well as the reflected lateral lines and the PMLs that define the boundaries of the domain (b) Main geometrical parameters of the multi-physical pickets; where a is the thickness of the absorbent material while $t$ is the thickness of the rigid element. The pickets are quarter wavelength resonators surrounded by absorbent material.

Although the model is very useful to select the action mechanism and the frequency to be attenuated, when the results are compared with those obtained experimentally in laboratory conditions, discrepancies appear. To validate the model, eliminating at the 
same time the discrepancies due to the finite size of the sample in the experimental measurements, a finite model with the same number of MP was implemented (Figure 3). The characteristics of this model are similar to those discussed above except for the dimensions of the domain and the surfaces conditions. Thus, as two rows with 6 pickets each have been used in such a way, it is not infinite along $\mathrm{X}$ direction. Furthermore, the domain is surrounded by PML to emulate the anechoic chamber conditions. The background field is used to introduce the plane wave in the domain.

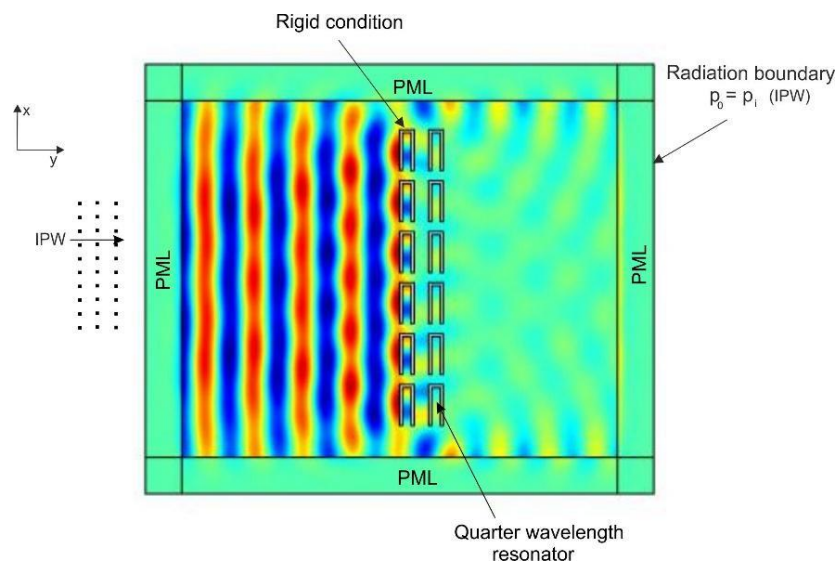

Figure 3.- Design finite model. Plan view (OXY, two dimensional case), which includes resonances and filter acoustics. One can see the OXY section of the multi-phenomena pickets quarter wavelength resonator, as well as the reflected lateral lines and the PMLs that define the boundaries of the domain. The incident plane wave travels in the direction OY

In both cases, the acoustic attenuation is evaluated by means of the insertion loss parameter (IL). This is defined as the difference between the sound level obtained with and without the barrier.

$$
I L=20 \log _{10}\left|\frac{P_{D}}{P_{I}}\right|(\mathrm{dB})
$$

where $\mathrm{P}_{\mathrm{D}}$ and $\mathrm{P}_{\mathrm{I}}$ are the direct and interferred sound pressures, respectively. 
As it has been mentioned in previous section, the sample consists on two rows of six pickets (Figure 4a). The pickets have a cavity in order to act as $L / 4$ resonator. In Figure $4 \mathrm{~b}$ a real picture of the picket is shown. Its height is $1.8 \mathrm{~m}$.

(a)

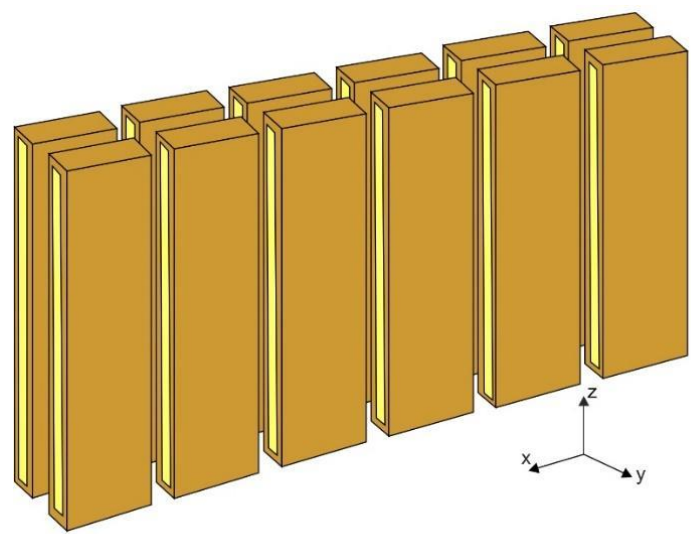

(b)

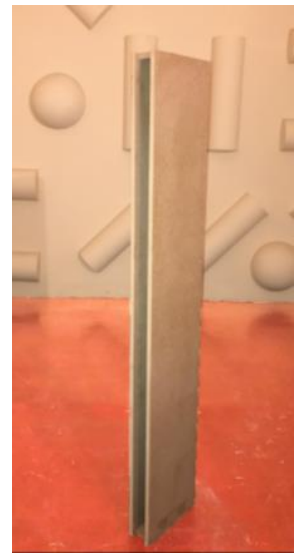

Figure 4.- (a) Outline of the picket structure that forms the acoustic barrier. The incident plane wave travels in the direction OY (b) A picture of the sample made of multi-phenomena picket.

\section{LABORATORY TESTING}

To experimentally analyse the influence of different parameters on the insertion loss of an acoustic barrier, tests can be carried out both in anechoic chamber $[7,9,10]$ and diffuse field in transmission chamber $[6,16,17]$. Now, to avoid the multiple reflections that take place in diffuse field masking the different phenomena that act on the acoustic barrier, it is useful to perform the measurements in an echo-free chamber. However, to standardise this barrier it will be necessary to characterise it according to standardised criteria as it is done in $[6,16,17]$. The standardised method has the advantage of allowing classification and comparison with other types of barriers.

To validate the numerical results obtained by means of our model, a set of experiments have been performed in an echo-free chamber of $8 \times 6 \times 3 \mathrm{~m}^{3}$, which simulate free field conditions (see Figure 5a). A prepolarised free-field 1/2" microphone Type 4189 B\&K 
located at $1.1 \mathrm{~m}$ from the sample (see Figure $5 \mathrm{~b}$ ) and a directional sound source GENELEC 8040A emitting continuous white noise, located $1 \mathrm{~m}$ behind the sample in order to consider the wave impinging on the sample as a plane wave, have been used throughout the experiments. The position of the microphone is controlled and varied by using a Cartesian robot (see Figure 5c). When the robotised system arrived at the measurement point it turned off and the microphone acquired the temporal signal. This signal is saved on the computer and then the frequency response of the measured sample is obtained using the Fast Fourier Transform (FFT). Using this microphone positioning system, the source-receiver line is always perpendicular to the length of the sample.

(a)

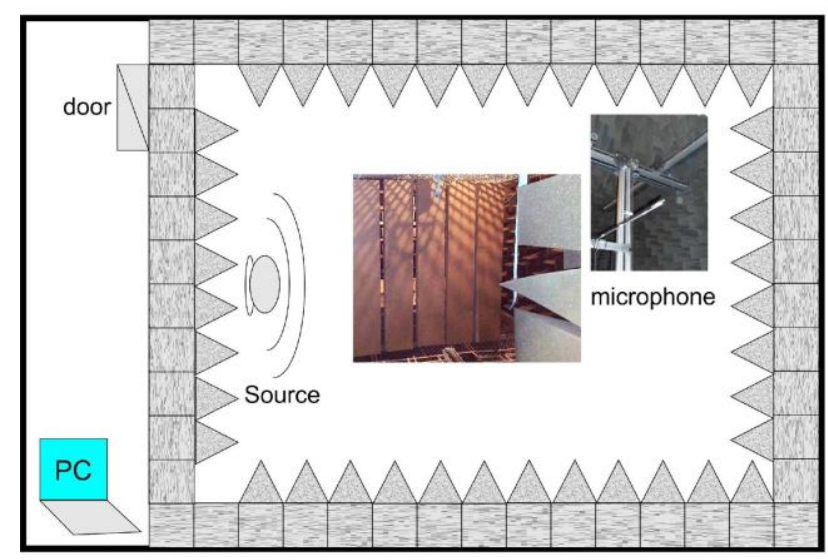

(b)

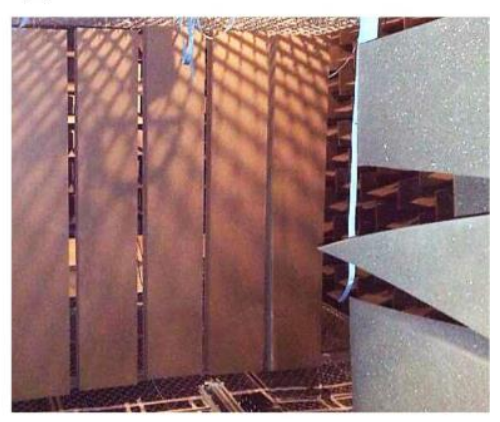

(c)

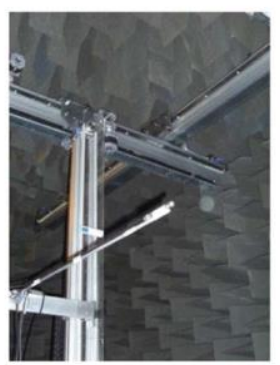

Figure 5.- (a) Scheme of the experimental set-up used. Chamber scheme and the arrangement of elements to carry out the measurements, (b) Detail of the OTAB sample picture, (c) Detail of the robot for positioning the microphone.

Although a directional sound source has been used in the measurements, a plane wave incidence has been considered in the FEM models developed. This approach has been 
used successfully over the last years, providing experimental results in good agreement with the numerical or analytical predictions obtained using plane wave incidence [6].

\section{RESULTS AND DISCUSSION}

Using the finite model described in previous sections, the IL (see eq. 1) for a quarter wavelength resonator pickets has been analysed and compared with experimental measures under controlled conditions. The geometric characteristics of the OTAB have already been described in section 2 . The resonators have been tuned to the frequency of $330 \mathrm{~Hz}$ so that the effectiveness of the barrier is greater at low frequencies. In such a way, the frequency range between 100 and $1200 \mathrm{~Hz}$ has been analysed. In this range, taking into account the geometric characteristics of the pickets, a the resonance peak at $330 \mathrm{~Hz}$ and its harmonic at $990 \mathrm{~Hz}$ can be observed . In Figure 6 the IL (dB) spectrum has been presented for an OTAB barrier both numerically and experimentally as well as the numerical results for a traditional barrier. The dotted black line corresponds to the numerical result of a traditional barrier with the same width as the OTAB. The continuous blue line corresponds to the OTAB numerical results obtained with the finite model and finally, a continuous red line represents the data obtained experimentally.

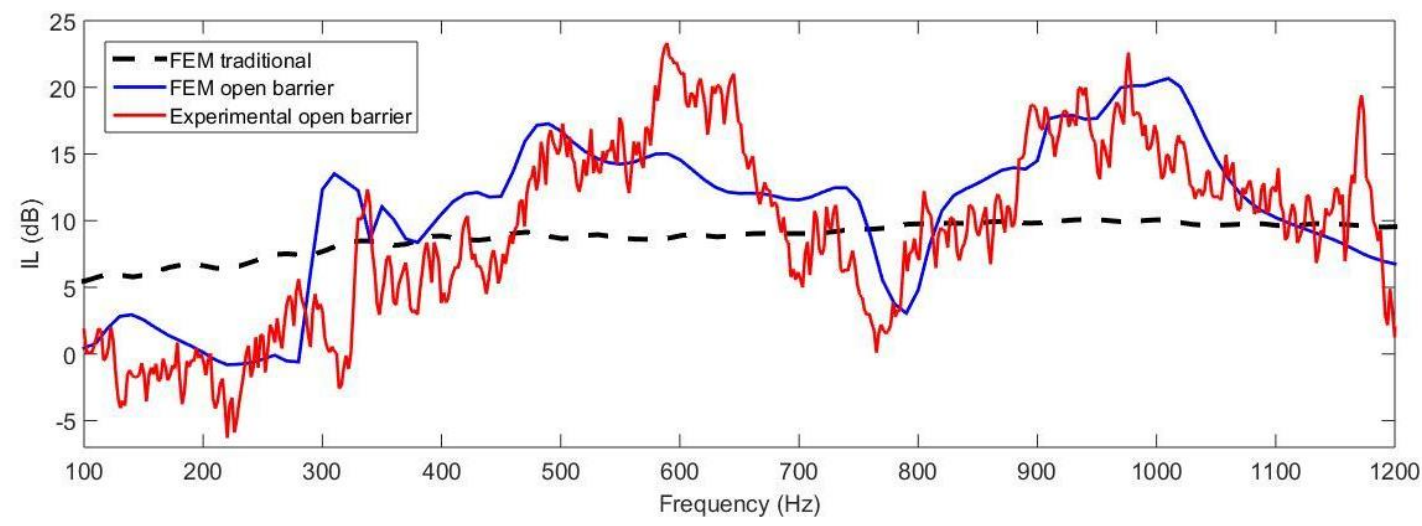

Figure 6.- IL spectra $(\mathrm{dB})$ : spectrum for a traditional barrier of the same width as the OTAB (Dotted black line), spectrum obtained numerically for the finite model of the OTAB (continuous blue line) and the spectrum obtained experimentally under controlled conditions for the OTAB (continuous red line). 
Note that in the zone between 330 and $990 \mathrm{~Hz}$ the OTAB presents a considerable improvement in the IL spectrum over the traditional one. This improvement is related to the resonances in the picket chamber. This effect is superimposed onto that obtained in the range of 400 to $700 \mathrm{~Hz}$, which is linked to the acoustic filter behaviour of the barrier. At the frequency of around $800 \mathrm{~Hz}$, a dip is observed in the IL spectrum. As previously explained, the acoustic barrier can be considered as a series of acoustic filters where the slits act as inlet / outlet apertures and the air gap between the pickets and the rows acts as an expansion chamber. In this type of filter, the IL reaches a minimum when the length of the expansion chamber or what is the same, the distance between the rows of pickets is a quarter of the wavelength, this is $\mathrm{k} \cdot \mathrm{d}_{\mathrm{ag}}=\pi / 2$, where $\mathrm{k}$ is the wavenumber [18].

On the other hand, the experimental and numerical results obtained for the OTAB agree. The diffraction at the top edge was not taken into account since the height of the pickets in the simulated barrier was considered infinite, so only the transmission through the barrier and the diffraction around the lateral edges were evaluated. In addition, viscous losses were not included, so these facts can cause discrepancies between simulated and measured results. These last results enable us to experimentally validate the model proposed.

Once the numerical model has been validated, and in order to introduce new noise mitigation mechanisms, new picket designs are proposed. In order to visualize with more clarity the effects that these mechanisms introduce, the ideal 2D compact model is used. Obviously, the results obtained with the $2 \mathrm{D}$ ideal model are maximised due to the fact that the barrier is considered infinitely long. In Figure 7a the IL spectrum obtained for the case of two rows of resonant pickets is represented. This model represents an infinite barrier in the OX direction as explained in section 2. The functioning of the resonators at $330 \mathrm{~Hz}$ and $990 \mathrm{~Hz}$ and the acoustic filter behaviour as mentioned in the analysis of the 
experimental results, are shown in Figure 6. Obviously, these effects are much better appreciated here and that is why this model is going to be used for the analysis of the new picket designs.

Three new picket designs are presented called Tuned-N. In all cases the dimensions and shape of the pickets have remained constant. For the first, called Tuned 1, the resonant cavities designed at $330 \mathrm{~Hz}$ have also been maintained and part of the rigid block has been replaced by absorbent material. The thickness of the absorber is $a$, and mineral wool material has been used as absorbent material with flow resistance $\mathrm{R}=23,000$ Pa.s.m- ${ }^{2}$. This material has modelled as a porous material using the Delany-Bazley model [14] implemented in COMSOL, as explained in section 2 . The rigidity of the picket is provided by a thin layer of thickness $t$. The result of the IL spectrum $(\mathrm{dB})$ in the study range frequency of this work, is shown in Figure $7 b$, where the designed resonators can be seen in the inset. It can be observed how the resonance peaks are maintained at $330 \mathrm{~Hz}$ and $990 \mathrm{~Hz}$ whilst the absorbing material introduces an extra attenuation from $400 \mathrm{~Hz}$ into the spectrum [8]. The effect of the absorber translates into a more homogeneous spectrum, that is, the resonance peaks fall at the expense of greater uniformity over a wide range of frequencies.

The second design, called Tuned 2, consists of quarter wavelength resonators tuned to two different frequencies. In such a way, two cavity lengths co-exist in the model. The rigid structure of the pickets has a thickness a $+\mathrm{t}$ like the initial design and no absorbent material is used. In order to achieve better performance of the barrier in the low frequencies range, the resonators of the first row of the barrier have been tuned to a frequency of $400 \mathrm{~Hz}$ while those of the second one have been maintained at $330 \mathrm{~Hz}$. The result of the IL spectrum $(\mathrm{dB})$ in the frequency range under study is shown in Figure 7c, where the designed resonators can be seen in the inset. It is possible to observe the two 
resonance peaks at low frequencies $330 \mathrm{~Hz}$ and $400 \mathrm{~Hz}$. With this design, a considerable level of attenuation at low frequencies has been achieved.

Finally, the third design called Tuned 3 is analysed, which is obtained modifying the Tuned 2 design by replacing the rigid part of the pickets with an absorbent part of width $a$ until completing the original size with a rigid thin layer of thickness $t$. Rock wool material has been used with flow resistance $\mathrm{R}=23,000 \mathrm{~Pa} \cdot \mathrm{s} . \mathrm{m}^{-2}$. We have modeled the rock wool as a material using the Delany-Bazley model [14] implemented in COMSOL, as discussed above. The result of the IL spectrum $(\mathrm{dB})$ in the study frequency range of this work, is shown in Figure 7d, where the designed resonators can be seen in the inset. It can be seen how the resonance peaks are maintained at $330 \mathrm{~Hz}$ and $990 \mathrm{~Hz}$ while the absorbing material introduces more attenuation from $400 \mathrm{~Hz}$, in the spectrum. The effect of the absorbent translates into a more homogeneous spectrum. It is observed that in the frequency range from 300 to $1000 \mathrm{~Hz}$, results are obtained that considerably improve those obtained with a traditional barrier of the same dimensions as the OTAB, making the latter competitive.
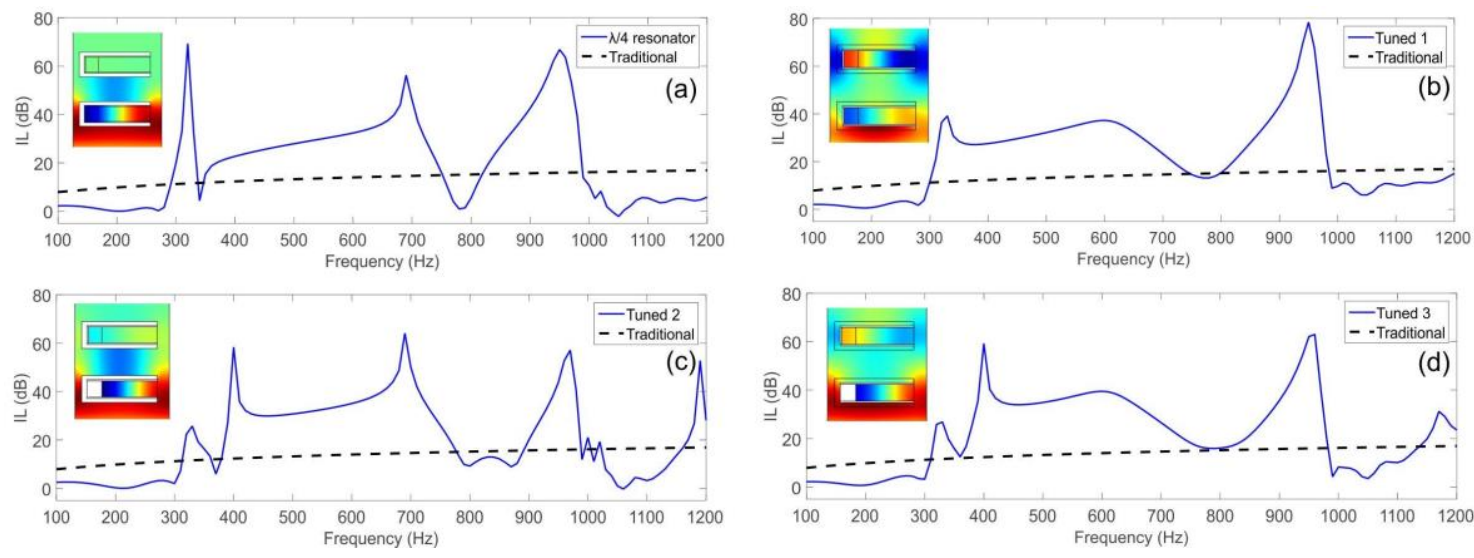

Figure 7.- IL spectra $(\mathrm{dB})$ : spectrum obtained with the ideal numerical model of the OTAB (continuous blue line), spectrum for a traditional barrier of the same width as the OTAB for all designs (Dotted black line). 
The variation of the absorbing material and therefore the value of the flow resistance has a direct influence on the attenuation capacity of the barrier. As shown in Figure 8, increasing the flow resistance increases the IL of the barrier. However, this increase in attenuation is small because the amount of absorbent that has been used in this type of device is small.

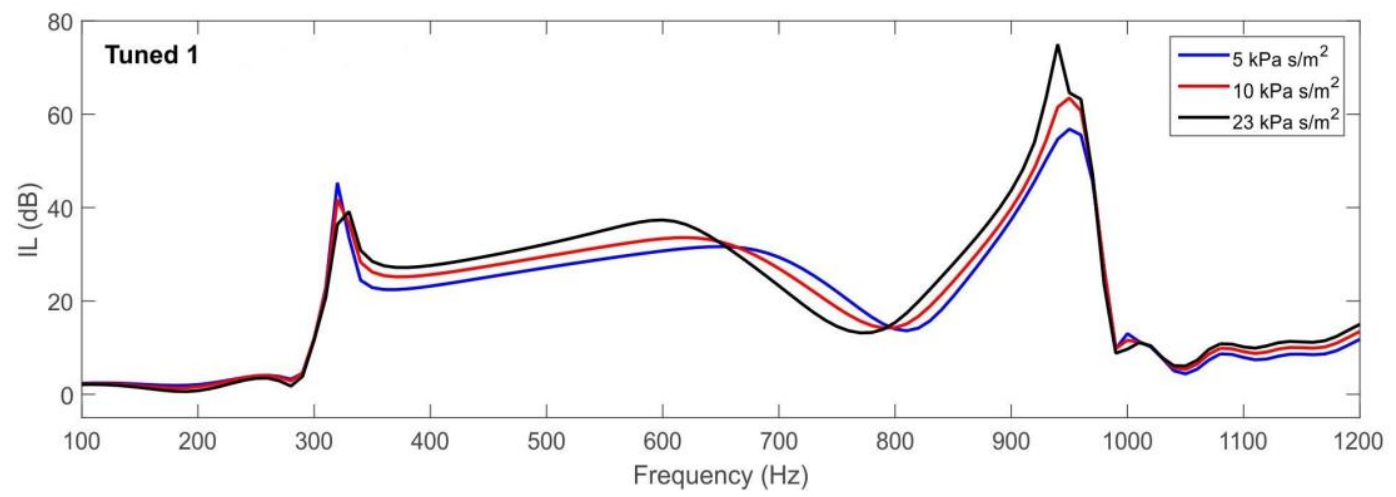

Figure 8.- IL spectra (dB): spectrum for different values of the flow resistance for a Tuned 1 case.

On the other hand, another configurable parameter is the distance between rows $\left(\mathrm{d}_{\mathrm{ag}}\right)$. In this case, it is well known that the wider the barrier, the more effective it is. Although in this case part of the width corresponds to air, this fact is still fulfilled at the frequencies for which the barrier has been tuned (330, 400 and $990 \mathrm{~Hz})$ as is shown in Figure 9. In addition, it can be observed that the attenuation peak located between 400-990 Hz moves towards high frequencies as $\mathrm{d}_{\mathrm{ag}}$ increases. This fact is explained as a destructive interference between the propagating and evanescent waves generated [9]. 


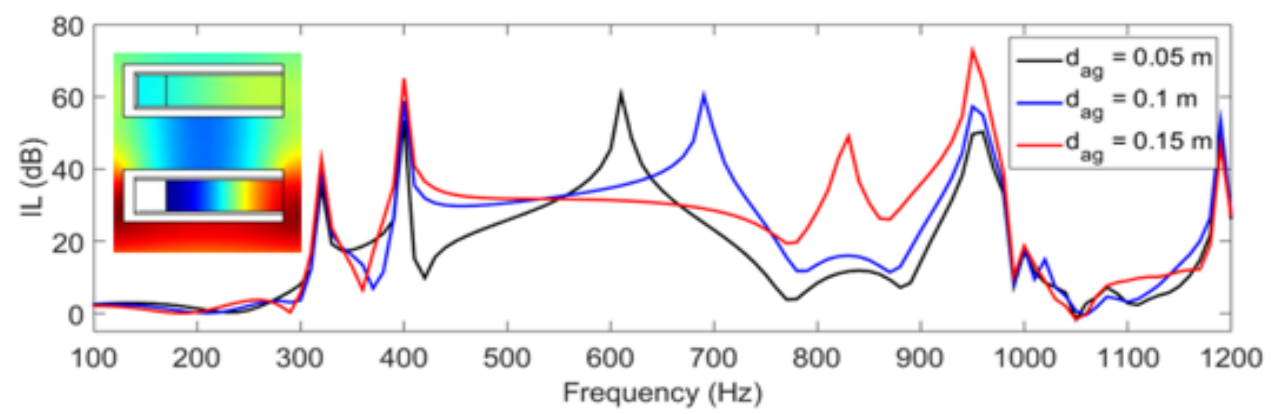

Figure 9. IL spectra (dB): spectrum for different values of distance between rows, $d_{a g}$, for a Tuned 2 case

\section{CONCLUSIONS}

An application of a model to design OTAB formed by multi-phenomena pickets embedded in air, taking into account the complex acoustic phenomenology that appears in the real use of this new kind of barrier, is presented in this paper. OTAB can be used in noise control in buildings to reduce the most important type of noise that appears in cities, i.e. transport noise, for aesthetic and technological reasons due to the fact that these barriers are versatile enough to be designed for specific noises. OTAB are technologically advanced and may become competitive with respect to traditional barriers. In order to design an OTAB for noise control, the versatile numerical model presented can be profitably used. The model is based on the idea of tuneability and allows the analysis of each one of the noise control mechanisms involved independently (resonances and acoustic filter) and, at the same time, can be used to find the best solution for each case without increasing the computational cost, selecting the range of frequencies in which the designer wants each noise control mechanism to work. The numerical simulations presented, supported by accurate experimental results allow the model to be validated. Moreover, the model allows the inclusion of any new noise control mechanism desired. 
This work was financially supported by the Spanish Ministry of Science and Innovation through project MAT2010-16879.

\section{REFERENCES}

[1] Watts, G, Acoustic performance of parallel traffic noise barriers. Applied Acoustics 47, 95-119, (1996).

[2] Watts, G, Godfrey, N, Effects on roadside noise levels of sound absorptive materials in noise barriers. Applied Acoustics 58, 385-402, (1999).

[3] Alfredson, R, Du, X, Special shapes and treatment for noise barriers. Proceedings of Internoise 95, pp. 381-384, (1995).

[4] Garai, M, Guidorzi, P, Sound reflection measurements on noise barriers in critical conditions, Building and Environment 94 (2015) 752-763.

[5] Arenas, JP, Potential problems with environmental sound barriers when used in mitigating surface transportation noise, Sci. Total Environ 405 (2008) 173-179.

[6] Castiñeira-Ibáñez, S., Rubio, C., Romero-García, V., Sánchez-Pérez, J.V., García-Raffi, L.M., Design, Manufacture and Characterization of an Acoustic Barrier Made of MultiPhenomena Cylindrical Scatterers Arranged in a Fractal-Based Geometry, Archives of Acoustics, 37(4), 455-462, (2012).

[7] Castiñeira-Ibáñez, S; Rubio, C; Sánchez-Pérez, J.V., Environmental noise control during its transmission phase to protect buildings. Design model for acoustic barriers based on arrays of isolated scatteres, Building and Environment, 93, 179-185, (2015).

[8] Van Campen /Bayards, https://vancampenbayards.com/en/projects/363-a2-noise-barrier-pipesbarrier-eindhoven. [24/11/2017]

[9] Rubio, C, Candelas, P, Belmar, F, Gomez-Lozano, V, Uris, A, Subwavelength slit acoustic metamaterial barrier, J. Phys. D.: Appl. Phys. 18 (2015) 395501-395510. 
[10] Romero-García V., Sánchez-Pérez J. V. and García-Raffi L. M., Tunable wideband bandstop acoustic filter based on two-dimensional multiphysical phenomena periodic systems. J. Appl. Phys., 110 (2011) 014904.

[11] Wood RW. Anomalous diffraction gratings. Phys. Rev. 48(12), 928-936, (1935).

[12] Akiyama K, Takano K, Abe Y, Tokuda Y, Hangyo M. Optical transmission anomalies in a double-layered metallic slit array. Optic Express ,18, 17876-17882, (2010)

[13] Berenguer J., A Perfectly Matched Layer for the absorption of Electromagnetic Waves, J. Comput. Phys., 114 (1994) 185.

[14] Delany ME, Bazley EN. Acoustical properties of fibrous absorbent materials. Applied Acoustics, 3, 105-116, (1970).

[15] Field CD, Fricke FR, Theory and Applications of Quarter-wave Resonators: A Prelude to Their Use for Attenuating Noise Entering Buildings Through Ventilation Openings. Applied Acoustics, 53, 117-132, (1998).

[16] Garcìa-Chocano VM, Sánchez Dehesa J, Optimum control of broadband noise by arrays of cylindrical units made of a recycled materials. Applied Acoustics, 74,58-62, (2013).

[17] Morandi F Miniaci M, Marzani A, Barbaresi L, Garai M, Standardised acoustic characterisation of sonic crystals noise barriers: Sound insulation and reflection properties, Applied Acoustics, 114,294-306, (2016).

[18] Kinsler, L. E., Frey, A. R., Coppens, A. B., \& Sanders, J. V. Fundamentals of acoustics. $4^{\text {th }}$ Edition, John Wiley \& Sons, 2000, Chapter 10. 


\section{FIGURES CAPTIONS}

Figure 1. Schematic diagram of a basic subwavelength slit acoustic barrier.

Figure 2.- Design of the ideal numerical model: (a) Plan view (OXY, two dimensional case), which includes resonances and acoustic filter. One can see the OXY section of the multi-phenomena pickets, as well as the reflected lateral lines and the PMLs that define the boundaries of the domain (b) Main geometrical parameters of the multi-physical pickets; where a is the thickness of the absorbent material while $t$ is the thickness of the rigid element. The pickets are quarter wavelength resonators surrounded by absorbent material.

Figure 3.- Design finite model. Plan view (OXY, two dimensional case), which includes resonances and filter acoustics. One can see the OXY section of the multi-phenomena pickets quarter wavelength resonator, as well as the reflected lateral lines and the PMLs that define the boundaries of the domain. The incident plane wave travels in the direction OY.

Figure 4.- (a) Outline of the picket structure that forms the acoustic barrier. The incident plane wave travels in the direction OY (b) A picture of the sample made of multiphenomena picket.

Figure 5.- (a) Scheme of the experimental set-up used. Chamber scheme and the arrangement of elements to carry out the measurements, (b) Detail of the OTAB sample picture, (c) Detail of the robot for positioning the microphone.

Figure 6.- IL spectra $(\mathrm{dB})$ : spectrum for a traditional barrier of the same width as the OTAB (Dotted black line), spectrum obtained numerically for the finite model of the OTAB (continuous blue line) and the spectrum obtained experimentally under controlled conditions for the OTAB (continuous red line) 
Figure 7.- IL spectra $(\mathrm{dB})$ : spectrum obtained with the ideal numerical model of the OTAB (continuous blue line), spectrum for a traditional barrier of the same width as the OTAB for all designs (Dotted black line).

Figure 8.- IL spectra $(\mathrm{dB})$ : spectrum for different values of the flow resistance for a Tuned 1 case.

Figure 9. IL spectra $(d B)$ : spectrum for different values of distance between rows, $d_{a g}$, for a Tuned 2 case 